\title{
An analysis of a class of telecommunications models
}

\author{
H.R. Gail ${ }^{\mathrm{a}, *}$, S.L. Hantler ${ }^{\mathrm{a}}$, A.G. Konheim ${ }^{\mathrm{b}}$, B.A. Taylor ${ }^{\mathrm{c}}$ \\ ${ }^{\text {a }}$ IBM Thomas J. Watson Research Center, Yorktown Heights, NY 10598, USA \\ ${ }^{\mathrm{b}}$ University of California, Santa Barbara, CA 93106, USA \\ ${ }^{\mathfrak{c}}$ University of Michigan, Ann Arbor, MI 48109, USA
}

\begin{abstract}
In this short note we outline a general method for characterizing the ergodicity and computing performance measures for a large class of telecommunications models. We also point out errors that have appeared in the literature when using the transform method to analyze such models.
\end{abstract}

Keywords: M/G/1 type Markov chain; Eigenvalue; Generating function; Asynchronous transfer mode; Multiplexor

\section{Introduction}

The transform method frequently has been used in the modeling and analysis of certain telecommunications systems. That is, to determine the equilibrium probabilities of the Markov chain that describes the behavior of such models, it is common to study an equivalent set of generating function equations. This technique involves finding singularities of an analytic matrix valued function inside the closed unit disk, and using these singularities to obtain equations for a finite set of boundary probabilities. Arguments usually involving Rouché's Theorem are used to count the zeros of the determinant of the matrix function. However, we show in Section 3 that applications of theorems from complex analysis that have appeared in the literature are often incorrect. We then present in Section 4 a correct argument that determines the number and location of the zeros. Conditions that describe when the chain is ergodic, null recurrent or transient are also given. We note that although these models appear to have different structures, they are all $M / G / 1$ type Markov chains with essentially identical transition matrices. Their analysis can be handled by a single argument, which we give below.

\footnotetext{
${ }^{*}$ Corresponding author.
} 


\section{M/G/1 type Markov chains}

The Markov chain governing the behavior of many telecommunications models is a particular example of the following class of chains. A discrete-time Markov chain $\mathscr{E}$ with states $(i, j)$, $i=0,1, \ldots, j=0, \ldots, M$ where $M$ is a nonnegative integer, is said to be of $\mathrm{M} / \mathrm{G} / 1$ type if its transition matrix is of the form

$$
\mathscr{P}=\left[\begin{array}{ccccccc}
b_{0,0} & b_{0,1} & \cdots & b_{0, N-1} & b_{0, N} & b_{0, N+1} & \cdots \\
\vdots & \vdots & \ddots & \vdots & \vdots & \vdots & \ddots \\
b_{N-1,0} & b_{N-1,1} & \cdots & b_{N-1, N-1} & b_{N-1, N} & b_{N-1, N+1} & \cdots \\
a_{0} & a_{1} & \cdots & a_{N-1} & a_{N} & a_{N+1} & \ldots \\
0 & a_{0} & \cdots & a_{N-2} & a_{N-1} & a_{N} & \ldots \\
\vdots & \vdots & \ddots & \vdots & \vdots & \vdots & \ddots
\end{array}\right] .
$$

The Markov chain $\mathscr{C}$ is assumed to be irreducible. The entries $b_{i k}, a_{k}, i=0, \ldots, N-1, k=0$, $1, \ldots$, are $(M+1) \times(M+1)$ matrices, and $N$ is a positive integer. The first coordinate of $(i, j)$ is called the level of the state, while the second coordinate is called the phase or stage of the state. States with a level of at least $N$ constitute the homogeneous part of the chain, while states with a level of at most $N-1$ are called boundary states. An extensive theory of these chains based on an algorithmic treatment has been developed by M.F. Neuts [11]. Another classical approach to their study is through the use of generating functions, and this method is fully developed in [2]. We now briefly review this latter approach based on techniques from complex analysis.

Our interest is in determining the stationary probabilities of the chain $\mathscr{C}$. That is, we seek $1 \times(M+1)$ vectors $\pi_{i}, i=0,1, \ldots$, where $\pi=\left[\pi_{0}, \pi_{1}, \ldots\right] \in l^{1}$, which satisfy $\pi=\pi \mathscr{P}$. Define the vector generating function corresponding to the homogeneous states

$$
G(z)=\sum_{i=N}^{\infty} \pi_{i} z^{i-N}
$$

and also define the generating functions

$$
a(z)=\sum_{k=0}^{\infty} a_{k} z^{k}
$$

and, for $i=0, \ldots, N-1$,

$$
b_{i}(z)=\sum_{k=0}^{\infty} b_{i, k} z^{k} .
$$

Transforming the balance equations yields

$$
G(z)\left[z^{N} I-a(z)\right]+\sum_{i=0}^{N-1} \pi_{i}\left[z^{i} I-b_{i}(z)\right]=0 .
$$

An equivalent equation involving the probability generating function

$$
\pi(z)=\sum_{i=0}^{\infty} \pi_{i} z^{i}=z^{N} G(z)+\sum_{i=0}^{N-1} \pi_{i} z^{i}
$$


is given by

$$
\pi(z)\left[z^{N} I-a(z)\right]+\sum_{i=0}^{N-1} \pi_{i}\left[z^{i} a(z)-z^{N} b_{i}(z)\right]=0 .
$$

Since $\pi \in l^{1}$, the unknown vector function $G(z)$ (or $\pi(z)$ ) is analytic in the open unit disk and continuous in the closure. The usual method employed to solve (5) or (7) is to find all singularities of the matrix valued function

$$
\Delta(z)=z^{N} I-a(z)
$$

in the closed unit disk and use them to find linear equations involving the probability vectors $\pi_{i}, i=0, \ldots, N-1$, associated to the boundary states. The solution of these equations, normalized by conservation of probability, yields the boundary probabilities, and hence from (5) or (7) the stationary probabilities $\pi_{i}, i=0,1, \ldots$ Although this approach is straightforward, there are many technical difficulties that must be resolved in order for it to be carried out rigorously. A second problem of interest is to characterize in terms of the matrices $a_{k}$ and $b_{i k}$ when the chain is ergodic, null recurrent or transient.

\section{Telecommunications Models}

A particular class of $\mathrm{M} / \mathrm{G} / 1$ type Markov chains has appeared recently to model the behavior of switches with multiple types of traffic (voice, data, video, multimedia) [4-10,12,1417]. The following model of a voice/data multiplexor appears in $[9,12]$. Time is divided into slots with $N$ slots comprising a frame. There are at most $M$ voice connections actively transmitting during a frame, and such connections can become active or inactive only at the beginning of a frame. The number of active voice connections per frame is governed by an $M+1$ state Markov chain with an irreducible, aperiodic transition matrix $Q=\left[q_{s s^{\prime}}\right], s$, $s^{\prime}=0, \ldots, M$. Data packets are one slot in length and have an arrival process with generating function $R(z)=\sum_{k=0}^{\infty} r_{k} z^{k}$. Packets that arrive during a frame can only be transmitted in subsequent frames, and there is an infinite buffer for data packets. Voice has priority over data, in that all active voice connections occupy one slot during a frame. Let $u_{n}$ be the number of buffered data packets at the beginning of the $n$th frame, $v_{n}$ the number of active voice connections at the beginning of the $n$th frame, and $w_{n}$ the number of data packets that arrive during the $n$th frame. Then $\left(u_{n}, v_{n}\right)$ is a two-dimensional Markov chain with states $(i, j), i=0$, $1, \ldots, j=0, \ldots, M$, the behavior of which is represented by the equation

$$
u_{n+1}=\left(u_{n}+v_{n}-N\right)^{+}+w_{n} .
$$

Here we use the notation $X^{+}=\max (X, 0)$.

By (9) the probabilities of transitions from level $N+i$ to level $k+i$ are identical for $i=0$, $1, \ldots$, so $\mathscr{P}$ is the transition matrix of an $\mathrm{M} / \mathrm{G} / 1$ type Markov chain. To find the entries of $\mathscr{P}$, 
consider a transition from level $N$, and note that if $u_{n}=N$ then $u_{n+1}=v_{n}+w_{n}$. Now a transition from state $(N, s)$ to state $\left(s+w, s^{\prime}\right)$ takes place with probability $q_{s s^{\prime}} r_{w}$, so that

$$
a(z)=R(z)\left[\begin{array}{cccc}
q_{00} & q_{01} & \ldots & q_{0 M} \\
z q_{10} & z q_{11} & \ldots & z q_{1 M} \\
\vdots & \vdots & \ddots & \vdots \\
z^{M} q_{M 0} & z^{M} q_{M 1} & \ldots & z^{M} q_{M M}
\end{array}\right] .
$$

The matrix functions $b_{i}(z), i=0, \ldots, N-1$, are also easy to obtain as

$$
b_{i}(z)=R(z)\left[\begin{array}{cccc}
q_{00} & q_{01} & \ldots & q_{0 M} \\
\vdots & \vdots & \ddots & \vdots \\
z q_{N-i+1,0} & z q_{N-i+1,1} & \ldots & z q_{N-i+1, M} \\
\vdots & \vdots & \ddots & \vdots \\
z^{M-N+i} q_{M 0} & z^{M-N+i} q_{M 1} & \ldots & z^{M-N+i} q_{M M}
\end{array}\right] \text {, }
$$

with $b_{i}(z)=R(z) Q$ for $0 \leq i \leq N-M$. We may write $a(z), b_{i}(z)$, in terms of the diagonal matrices

$$
D_{i}(z)=\operatorname{diag}\{\underbrace{1, \ldots, 1,}_{N-i+1} z, \ldots, z^{M-N+i}\}
$$

as $a(z)=R(z) D_{N}(z) Q$ and $b_{i}(z)=R(z) D_{i}(z) Q, i=0, \ldots, N-1$. The generating function equation (7) is then

$$
\pi(z)\left[z^{N} I-R(z) D_{N}(z) Q\right]=\sum_{i=0}^{N-1} \pi_{i} R(z)\left[z^{N} D_{i}(z)-z^{i} D_{N}(z)\right] Q .
$$

The equation derived in [4] is slightly different, since data packets do not have to wait until subsequent frames to be transmitted in the model studied there. Instead, a packet may be served in any slot after the one in which it arrives. In this case, the matrix function $\Delta(z)=z^{N} I-R(z) D_{N}(z) Q$ is the same as before since (9) still holds when $u_{n}$ is at least $N$, but the $b_{i}(z)$ are different.

The first problem to be addressed is the determination of the number and location of the zeros of det $\Delta(z)$ in the closed unit disk. The following argument is given in [4]. Write

$$
\operatorname{det} \Delta(z)=\operatorname{det}\left[z^{N} I-R(z) D_{N}(z) Q\right]=\prod_{j=0}^{M}\left[z^{N}-R(z) \lambda_{j}(z)\right],
$$

where $\lambda_{j}(z), j=0, \ldots, M$, are the $M+1$ eigenvalues of $D_{N}(z) Q$ and where $\left|\lambda_{j}(z)\right|<1$ for $|z|<1$. If the $\lambda_{j}(z)$ were analytic in the open unit disk as asserted in Lemma 4.2 of [4], one might apply Rouché's Theorem to the individual factors $z^{N}-R(z) \lambda_{j}(z)$ to find the number 
and location of the zeros of det $\Delta(z)$ in the unit disk. The problem with this argument is that the $\lambda_{j}(z)$ are not, in general, even continuous functions.

Example 1. Consider the simplest case $M=1$ ( $N$ arbitrary), and let

$$
Q=\left[\begin{array}{cc}
q_{0} & 1-q_{0} \\
1-q_{1} & q_{1}
\end{array}\right],
$$

where $0<q_{0}<q_{1}<1$ and $q_{1} \neq 1-q_{0}$. For a fixed $z$, the eigenvalues of $D_{N}(z) Q$ are the complex numbers $\lambda=\lambda(z)$ that satisfy the equation

$$
\operatorname{det}\left\{\lambda I-D_{N}(z) Q\right\}=\lambda^{2}-\left(q_{0}+z q_{1}\right) \lambda-z\left(1-q_{0}-q_{1}\right)=0 .
$$

The roots of this equation are continuous functions in the unit disk if and only if the two zeros, $\xi_{0}$ and $\xi_{1}$, of the discriminant

$$
\delta(z)=q_{1}^{2} z^{2}+\left[2 q_{0} q_{1}+4\left(1-q_{0}-q_{1}\right)\right] z+q_{0}^{2}
$$

either coincide or lie outside the unit disk. However, $\xi_{0} \xi_{1}=\left(q_{0} / q_{1}\right)^{2}<1$, and thus at least one of $\xi_{0}$ and $\xi_{1}$ is inside the unit disk. Further, $-\left(\xi_{0}+\xi_{1}\right)=\left[2 q_{0} q_{1}+4\left(1-q_{0}-q_{1}\right)\right] / q_{1}^{2}$, so if the two roots coincide then

$$
-\xi_{0}=\frac{q_{0}}{q_{1}}-2 \frac{1-\left(1-q_{0}\right) / q_{1}}{q_{1}}, \quad \xi_{0}^{2}=\frac{q_{0}^{2}}{q_{1}^{2}} .
$$

This can happen if and only if $q_{1}=1$ or $q_{1}=1-q_{0}$, which is ruled out by assumption. Hence, the eigenvalues are not continuous functions in the unit disk. What is actually demonstrated in Lemma 4.2 of [4] is only that the leading term in the Puiseux series at the origin for $\lambda_{j}(z)$ has integral exponent.

In Proposition 4.1 of [5] it is noted that if the eigenvalues $\lambda_{j}(z)$ are all distinct then they are analytic, which is true. However the authors also assert that if the eigenvalues are not distinct, then the input parameters can be perturbed to make them so. That is, every set of input parameters is a limit point of input parameters for which the eigenvalues are analytic, which is false. Note that there is no way to perturb the input parameters $q_{0}$ and $q_{1}$ in the above example to obtain analytic eigenvalues.

In addition, Rouché's Theorem is applied in Proposition 4.1 of [5] to an equation $z^{N}=\lambda_{j}(z)$ with $\left|\lambda_{j}(z)\right| \leq 1$ to deduce that it has $N$ roots in $|z| \leq 1$. The example $z^{K}, K>N$, illustrates that the requirement of strict inequality on the unit circle to find roots in the open disk cannot be replaced by nonstrict inequality to find roots in the closed disk when using Rouché's Theorem.

A slightly different model than the one discussed above has appeared in the sequence of papers $[6-8,10,14-16]$. The two-dimensional Markov chain that is studied is governed by the simpler equation

$$
u_{n+1}=\left(u_{n}+v_{n}-N\right)^{+},
$$


where $v_{n}$ is the amount of traffic arriving during the $n$th frame, and $u_{n}$ is the amount of buffered traffic at the beginning of the $n$th frame (an infinite buffer is assumed). Here $v_{n}$ may consist of many different types of traffic (e.g. multimedia), and each type may have distinct statistical properties. In this case (see [13] and Section 6.2.B of [11]), the traffic matrix decomposes into the Kronecker product of matrices associated with the individual traffic types, and this decomposition can be exploited in the analysis. However, it is clear that (11) defines a chain of $\mathrm{M} / \mathrm{G} / 1$ type of the previous form with $R(z)=1$ (note that $M \geq N$ may hold), and thus the above comments again apply. For example, the generating function equation for $\pi(z)$ is

$$
\pi(z)\left[z^{N} I-D_{N}(z) Q\right]=\sum_{i=0}^{N-1} \pi_{i}\left[z^{N} D_{i}(z)-z^{i} D_{N}(z)\right] Q
$$

which is easily seen to be equivalent to the main equation (2.12) in [6], (22) in [10], (2.12) in [7], (2.3) in [8], (9) in [14], (9) in [15] after some straight-forward manipulations. The assertion that the eigenvalues are analytic is again false, and, in fact, the $Q$ matrix given in Example 1 is the matrix studied in equation (3.1) of [6], (45) of [10], (3.1) of [7], (3.1) of [8], (46) of [15].

Additional errors appear in these papers. On page 2027 of [16] it is claimed that the infinite buffer equation (11) is the evolution equation for the previous model considered with a finite buffer. Further, balance equations for the infinite buffer case are said to hold for the finite buffer case (see equations (4)-(6) in [16]). Zeros of the generating function for the infinite buffer model are found on page 2028 of that paper using the above incorrect eigenvalue arguments, and they then are used to analyze the finite buffer model.

In equation (2.15) of [6], (26) of [10], (2.15) of [7], (2.5) of [8], it is claimed that the matrix $a(z)=D_{N}(z) Q$ can be diagonalized. However, Example 1 shows that this matrix is not diagonalizable in general. Also, in these papers, the left and right eigenvectors associated to the eigenvalues $\lambda_{j}(z)$ are determined, and it is asserted that they are analytic in the closed disk. In fact, equations (3.7) and (3.8) in [7] provide a counterexample to this assertion. On page 2169 of [8] it is stated that each $\lambda_{j}(z)$ is a different branch of an algebraic function, and all of them are analytic in the closed unit disk. Again, this is not true in general. Using zeros of these eigenvalues to obtain numerical results is also discussed in $[6-8,10,14,15]$. Since these functions may not even be continuous, it is not clear what sort of search procedure should be used to find their roots.

On page 1120 of [7] the following argument appears involving an eigenvalue $\lambda_{0}(z)$. It is stated that since $\lambda_{0}(1)=1, \lambda_{0}(0)=q_{0}$, and $\lambda_{0}^{\prime}(z) \neq 0$ for $0<|z|<1$, then by properties of conformal mapping $q_{0}<\left|\lambda_{0}(z)\right|<1$. First, as shown above $\lambda_{0}(z)$ is not in general differentiable, and it is not conformal. Further, the example $(1+z) / 2$ satisfies the above conditions, but clearly $1 / 2<|(1+z) / 2|<1$ does not hold in the punctured disk.

In [17] the results of [4] are generalized to arbitrary irreducible aperiodic matrices $Q$ (the assumptions in [4] include Poisson arrivals of voice connections and geometric distribution of length of connection). We will see below that the root count given in [17] is not correct for these generalizations. Further, the Markov chain $\mathscr{C}$ is not necessarily irreducible without additional assumptions on $Q$. These comments also apply to the model with evolution equation (11) studied in [6-8,10,14-16]. 
Example 2. Let $M=4, N=2$, and consider the irreducible aperiodic matrix

$$
Q=\left[\begin{array}{ccccc}
1 / 3 & 1 / 3 & 1 / 3 & 0 & 0 \\
0 & 0 & 0 & 1 & 0 \\
0 & 0 & 0 & 0 & 1 \\
1 & 0 & 0 & 0 & 0 \\
1 & 0 & 0 & 0 & 0
\end{array}\right]
$$

Then $\mathscr{C}$ is not irreducible, since $\{0,1,2\}$ constitutes an absorbing set of levels.

\section{Analysis of telecommunications models}

Determining the zeros of det $\Delta(z)$ in the closed unit disk is a nontrivial problem for irreducible $M / G / 1$ type Markov chains $\mathscr{C}$, with complications involving multiple zeros and zeros on the unit circle. In [2] it is shown that the number and location of the zeros depends on the structure of the matrix $a(1)=\sum_{k=0}^{\infty} a_{k}$. For the telecommunications model, the matrices $a(1)=b_{i}(1)=Q$ are irreducible, and the following is known in this case. The number, $g$, of distinct zeros of det $\Delta(z)$ on the unit circle satisfies $1 \leq g \leq(M+1) N$ when the chain $\mathscr{E}$ is irreducible. Further, $g$ has a probabilistic interpretation in terms of the changes in level for cycles between stages of $\mathscr{E}$ (see [2] for a precise interpretation). The zeros in the open unit disk are determined by the integer $g$ and the real number

$$
\gamma=\left.\frac{\mathrm{d}}{\mathrm{d} z} \operatorname{det} \Delta(z)\right|_{z=1},
$$

which is closely related to the drift in the homogeneous part of the chain. The next theorem is a consequence of Theorem 2 of [2].

Theorem 1. Suppose that $R(z)$ is continuously differentiable for $|z| \leq 1$ and that $a(1)$ is irreducible.

(i) If $\gamma>0$, then $\operatorname{det} \Delta(z)$ has exactly $(M+1) N-g$ zeros (counting multiplicities) in the open unit disk and simple zeros at the gth roots of unity on $|z|=1$.

(ii) If $\gamma<0$, then det $\Delta(z)$ has exactly $(M+1) N$ zeros (counting multiplicities) in the open unit disk and simple zeros at the gth roots of unity on $|z|=1$.

Suppose, in addition, that $R(z)$ is twice differentiable in the closed unit disk.

(iii) If $\gamma=0$, then det $\Delta(z)$ has exactly $(M+1) N-g$ zeros (counting multiplicities) in the open unit disk and zeros of multiplicity 2 at the gth roots of unity on $|z|=1$.

We will briefly sketch a proof of this theorem under the additional hypothesis that $R(z)$ is analytic in a neighborhood of $|z| \leq 1$. Without this hypothesis, more intricate technical arguments are required (a complete proof appears in [2]).

Proof. Define the matrix functions $\Delta(z, t)=z^{N} I-t a(z)$ indexed by the real parameter $0 \leq t \leq 1$. Clearly det $\Delta(z, 0)=z^{(M+1) N}$ has $(M+1) N$ zeros in the open unit disk and none on the unit circle. A computation shows that $\Delta(z, t)$ is a strictly diagonally dominant matrix for 
$0 \leq t<1,|z|=1$, and so it is nonsingular. Therefore, by the argument principle [1], the number of zeros of det $\Delta(z, t)$ in the open disk is a continuous integer-valued function of $t$. Thus det $\Delta(z, t)$ also has $(M+1) N$ zeros in the open disk and none on the boundary for $0 \leq t<1$. The proof is completed for $t=1$ by considering $\operatorname{det} \Delta(z, 1)$ at $z=1$, since the behavior is the same at all $g$ zeros on the unit circle because $z^{-(M+1) N}$ det $\Delta(z, t)$ is a function of $z^{g}$ [2]. First note that det $\Delta(1, t)>0$ for $0 \leq t<1$, since the determinant is nonzero for such $t$ and is positive when $t=0$. Using this, it is easy to show that the zero of det $\Delta(z, 1)$ at $z=1$ is the limit of real zeros of det $\Delta(z, t)$ from inside the unit disk as $t \rightarrow 1$ when $\gamma>0$, while it is the limit of real zeros from outside the disk when $\gamma<0$. For the remaining case, it is shown in [2] that $\mathrm{d}^{2} /\left.\mathrm{d} z^{2}(\operatorname{det} \Delta(z))\right|_{z=1}<0$ when $\gamma=0$, so that the determinant has a zero of order 2 at $z=1$, and it is the limit of real zeros from both inside and outside the unit disk.

In [4] the arrival process of voice connections is Poisson, and the duration of a connection has a geometric distribution. As a consequence, $z=1$ is the only zero of det $\Delta(z)$ on the unit circle, i.e. $g=1$. However, [17] attempted to generalize these results to cases where the arrival process was given by an arbitrary irreducible aperiodic matrix $Q$. In these cases, $g$ may be greater than 1 , even when $\mathscr{C}$ is irreducible. Thus the resulting theorems counting the zeros of det $\Delta(z)$ are incorrect. The same type of error occurs in $[15,16]$ for the model corresponding to equation (11).

Example 3: Consider the case $M=3, N=4$, of Eq. (9), and let

$$
Q=\left[\begin{array}{cccc}
0 & 1 / 2 & 1 / 2 & 0 \\
0 & 0 & 0 & 1 \\
1 & 0 & 0 & 0 \\
1 & 0 & 0 & 0
\end{array}\right],
$$

and $R(z)=\left(1+z^{4}\right) / 2$. Then $Q$ is irreducible and aperiodic, $\mathscr{C}$ is irreducible, $\gamma>0, g=2$, and $\operatorname{det} \Delta(z)$ has zeros on the unit circle at $z=1, z=-1$. On page 868 of [17], it is claimed that $z=1$ is the only zero of modulus 1 in this case.

Example 4. To find an example for Eq. (11), let $M=4, N=2$, and

$$
Q=\left[\begin{array}{ccccc}
1 / 3 & 1 / 3 & 1 / 3 & 0 & 0 \\
0 & 0 & 0 & 1 & 0 \\
0 & 0 & 0 & 0 & 1 \\
1 & 0 & 0 & 0 & 0 \\
1 / 2 & 0 & 0 & 0 & 1 / 2
\end{array}\right]
$$

Then $Q$ is irreducible and aperiodic, $\mathscr{C}$ is irreducible, $\gamma>0$ and $g=2$. Again on page 194 of [15] and page 2028 of [16] it is claimed that $z=1$ is the only zero on the unit circle.

A second question of interest is to characterize the ergodicity of the Markov chain $\mathscr{E}$. This depends on $\gamma$ and $R^{\prime}(1)$, the mean number of data packets that arrive during a frame. Although the correct condition was given in [4], it was based on the incorrect Lemma 4.2. The following is a consequence of Theorem 7 of [2]. 
Theorem 2. Suppose $a(1)$ is irreducible. The Markov chain $\mathscr{C}$ is transient if and only if $\gamma<0$. It is ergodic if and only if $\gamma>0$ and $R^{\prime}(1)<+\infty$.

The quantity $\gamma$ has a natural interpretation that allows the above characterization of ergodicity to be given in intuitive terms. Consider the equations

$$
\operatorname{adj}\{\Delta(z)\} \Delta(z)=\operatorname{det} \Delta(z) I=\Delta(z) \operatorname{adj}\{\Delta(z)\},
$$

where adj represents the classical adjoint matrix. Differentiating the left-hand equation and evaluating the result at $z=1$ yields

$$
\operatorname{adj}\{\Delta(1)\} \Delta^{\prime}(1)+\left.\frac{\mathrm{d}}{\mathrm{d} z} \operatorname{adj}\{\Delta(z)\}\right|_{z=1} \Delta(1)=\gamma I .
$$

Multiplying an $(M+1) \times(M+1)$ matrix on the right by the $(M+1) \times 1$ column vector 1 every entry of which is 1 simply adds the rows of the matrix. Now $\Delta(1)=I-a(1)$ has zero row sums because $a(1)$ is stochastic, so

$$
\operatorname{adj}\{\Delta(1)\} \Delta^{\prime}(1) \mathbf{1}=\gamma \mathbf{1}
$$

Since $\operatorname{det} \Delta(1)=0$, (14) shows that the rows and columns of $\operatorname{adj}\{\Delta(1)\}$ are left and right eigenvectors of $a(1)$ for the eigenvalue 1 . Further, since $a(1)$ is an irreducible stochastic matrix, it is well-known that the entries of adj $\{\Delta(1)\}$ are strictly positive (see page 75 of [3]). These facts imply that the rows of adj $\{\Delta(1)\}$ are all equal and are a positive multiple of $\eta$, the stationary probability vector of $a(1)$. That is,

$$
\operatorname{adj}\{\Delta(1)\}=c\left[\begin{array}{c}
\eta \\
\vdots \\
\eta
\end{array}\right],
$$

where $c>0$ and $\eta=\eta a(1)$. In addition, we have

$$
\Delta^{\prime}(1) \mathbf{1}=\left.\frac{\mathrm{d}}{\mathrm{d} z}\left[z^{N} I-a(z)\right]\right|_{z=1} \mathbf{1}=N \mathbf{1}-a^{\prime}(1) \mathbf{1} .
$$

Substituting (16) and (17) into (15) and using $\eta \mathbf{1}=1$ yields

$$
\gamma / c=N-\eta a^{\prime}(1) \mathbf{1},
$$

for a Markov chain of $M / G / 1$ type with an irreducible $a(1)$ matrix. The expression on the right-hand side of the above equation is the negative of the one step drift in levels for the homogeneous part of the chain.

For the telecommunications case, using $Q \mathbf{1}=\mathbf{1}$ we have

$$
a^{\prime}(1) \mathbf{1}=\left[\operatorname{diag}\{0,1, \ldots, M\}+R^{\prime}(1)\right] Q \mathbf{1}=\boldsymbol{h}+R^{\prime}(1) \mathbf{1},
$$

where $\boldsymbol{h}$ is the $(M+1) \times 1$ column vector $[0,1, \ldots, M]^{\mathrm{T}}$. Since $a(1)=Q, \eta$ is the stationary probability vector of the irreducible stochastic matrix $Q$. Thus from (18)

$$
\gamma / c=N-(\bar{v}+\bar{w}),
$$


where $\bar{v}=\sum_{j=0}^{M} j \eta_{j}$ is the mean number of active voice connections per frame and where $\bar{w}=R^{\prime}(1)$ is the mean number of arriving data packets per frame. The number of zeros of det $\Delta(z)$ and the ergodicity of the chain $\mathscr{C}$ can now be given in very natural probabilistic terms. For example, when $R^{\prime}(1)<+\infty$, the chain is ergodic, null recurrent, or transient if and only if the average amount of work arriving per frame, $\bar{v}+\bar{w}$, is less than, equal to, or greater than $N$, the number of slots per frame.

\section{Conclusions}

In this note we have given an analysis of a class of telecommunications models, and in the process we have corrected errors that have appeared in the literature. Specifically, we have determined the number and location of zeros of the determinant of a fundamental matrix function, which are used to obtain boundary probabilities of the Markov chain model. We have also given the stability condition for the chain, which has a natural probabilistic interpretation. We also wish to reiterate that the models used are special cases of Markov chains of $M / G / 1$ type, and in addition to the generating function approach there is a general algorithmic theory of these chains in the work of M.F. Neuts.

\section{References}

[1] L.V. Ahlfors, Complex Analysis (McGraw-Hill, New York, 2nd ed., 1966).

[2] H.R. Gail, S.L. Hantler and B.A. Taylor, Spectral analysis of $M / G / 1$ type Markov chains, IBM Research Report RC 17765, 1992.

[3] F.R. Gantmacher, Applications of the Theory of Matrices (Interscience, New York 1959).

[4] A.G. Konheim and M. Reiser, The moveable-boundary multiplexor stability and decomposability, in: O.J. Boxma, J.W. Cohen, H.C. Tijms (Eds.), Teletraffic Analysis and Computer Performance Evaluation (North-Holland, Amsterdam, 1986).

[5] R.H. Kwong and A. Leon-Garcia, Performance analysis of an integrated hybrid-switched multiplex structure, Perform. Eval. 4 (1984) 81-91.

[6] S.Q. Li, A general solution technique for discrete queueing analysis of multi-media traffic on ATM, INFOCOM '90 (1990) 1144-1155.

[7] S.Q. Li, A general solution technique for discrete queueing analysis of multi-media traffic on ATM, IEEE Trans. Comm. 39(7) (1991) 1115-1132.

[8] S.Q. Li, Generating function approach for discrete queueing analysis with decomposable arrival and service Markov chains, INFOCOM'92 (1992) 2168-2177.

[9] S.Q. Li and J.W. Mark, Performance of voice/data integration on a TDM switch, IEEE Trans. Comm. 33(12) (1985) $1265-1273$.

[10] S.Q. Li and H.D. Sheng, Discrete queueing analysis of multi-media traffic with diversity of correlation and burstiness properties, INFOCOM' 91 (1991) 368-381.

[11] M.F. Neuts, Structured stochastic Matrices of $M / G / 1$ Type and Their Applications (Marcel Dekker, New York, 1989)

[12] K. Sriram, P.K. Varshney and J.G. Shanthikumar, Discrete-time analysis of integrated voice/data multiplexers with and without speech activity detectors, IEEE J. Selected Areas Comm. 1(6) (1983) 1124-1132.

[13] A.M. Viterbi, Approximate analysis of time-synchronous packet networks, IEEE J. Selected Areas Comm. 4(6) (1986) 879-890. 
[14] Z. Zhang, A single server queue with finite-geometric-cluster arrival process, ICC '91 (1991) 1329-1334.

[15] Z. Zhang, Analysis of a discrete-time queue with integrated bursty inputs in ATM networks, Int. J. Digital Analog Comm. Systems 4 (1991) 191-203.

[16] Z. Zhang, Finite buffer discrete-time queues with multiple Markovian arrivals and services in ATM networks, INFOCOM' 92 (1992) 2026-2035.

[17] Z. Zhang and I. Rubin, Bounds on the mean system-size and delay for a moveable-boundary integrated circuit and packet switched communications channel, INFOCOM' 90 (1990) 866-873. 